\title{
Improving Cloud and Moisture Representation in Weather Prediction Model Analyses with Geostationary Satellite Information
}

\author{
Jordan J. Gerth ${ }^{1}$ \\ Department of Atmospheric and Oceanic Sciences \\ Cooperative Institute for Meteorological Satellite Studies \\ University of Wisconsin, Madison, Wisconsin
}

\begin{abstract}
This paper develops a methodology for an experiment with several parallel regional Weather Research and Forecasting (WRF) model simulations initialized with satellite-based retrievals. The intent is to clarify the impact of observations, in the form of retrievals, from the Geostationary Operational Environmental Satellite (GOES) Sounder on 12, 24, and 36-hour WRF model forecasts of precipitable water. Two experimental analyses are built from a CIMSS Regional Assimilation System (CRAS) pre-forecast spin-up. The CRAS assimilates precipitable water and cloud products derived from the GOES Sounder. An experimentation period between late September and early October 2011 found that the majority of impact in the experimental simulations compared to the control is recognized in the total precipitable water field over the first 12 hours.
\end{abstract}

\section{Introduction}

Water vapor is an important molecule in our atmosphere which has a profound impact on the dynamics and physics of the fluid earth system. Accurately assessing magnitudes and gradients of moisture in the troposphere, especially in the boundary layer, is an ongoing challenge. While in-situ observational data from surface stations and radiosondes paint a partial picture of the moisture distribution in the atmosphere, information collected from weather satellites is the only way to determine short-term changes in water vapor on spatial scales under a few hundred kilometers.

Many of the earth's most significant weather phenomena are a consequence of temperature and moisture gradients. The tropics and middle latitudes contain a substantial amount of water vapor, which condenses to produce clouds and precipitation. In order to better forecast the broad spectrum of diabatic weather processes, it is necessary to improve understanding of such processes on multiple spatial and temporal scales, from mesoscale convective systems (MCSs) to synoptic-scale mid-latitude weather systems, through their simulated evolution in numerical weather prediction (NWP) guidance. Essential to accurately resolving and parameterizing these phenomena as part of a forecast is incorporating satellite observations of cloud and water vapor into numerical models. The consistent use of these observations in real-time model simulations has the potential to improve predictions of storms and precipitation, a claim which is investigated here.

Since 1992, the development of the dynamics and physics within the Cooperative Institute for Meteorological Satellite Studies (CIMSS) Regional Assimilation System (CRAS) weather prediction model (http://cimss.ssec.wisc.edu/cras/) has been guided by the addition of satellite products into the assimilation pre-forecast (Aune 1994). During the pre-forecast, cloud and precipitable water $(\mathrm{PW})$ products from the twelve hours ahead of the initialization time are substantiated in the modeled atmosphere. These products are predominantly from the

\footnotetext{
${ }^{1}$ Funding for this research supplied in part by the Wisconsin Space Grant Consortium.
} 
Geostationary Operational Environmental Satellites (GOES) Imager and Sounder due to their relative temporal frequency. Polar-orbiting satellites, such as those equipped with a MODerate resolution Imaging Spectroradiometer (MODIS), can also be used where temporal frequency can be sacrificed in place of increased spatial and spectral resolution. The goal of the CRAS has been to show forecast improvement when additional satellite data sets are added to the traditional in-situ observations (R. Aune 2011, personal communication). In recent years, however, other modeling systems have grown in popularity as the development of the CRAS slowed. Despite this, the use of CRAS output gradually expanded into dozens of National Weather Service (NWS) forecast offices between 2006 and 2011. Forecaster comments reveal that the CRAS output continues to have a positive impact in certain forecast situations.

In contrast to the CRAS, the Weather Research and Forecasting (WRF) model (http://wrfmodel.org/) is a NWP model built from an increasingly popular collection of code for simulating atmospheric conditions at high spatial scales (Skamarock et al. 2008). The WRF model is a state-of-the-art mesoscale NWP tool which was developed to satisfy the needs of both operational forecasters in the field and atmospheric scientists in a research setting. This functionality allows the WRF model to be used both in scientific studies and for real-time prediction. NWS field offices across the United States are increasingly reliant on output from the WRF Environmental Modeling System (EMS), an end-to-end distributable for running the WRF locally and producing output (http://strc.comet.ucar.edu/wrfems/).

Since the WRF has been widely adopted for forecast applications, with improvements to its dynamical cores and physical packages continuing through the present time, it is an ideal platform for observation impact studies because of the applicability to numerous real-time users. Obtaining a better solution via a more accurate set of initial conditions is a long-standing tenet of NWP mathematics. The CRAS pre-forecast methodology remains a viable source of initial conditions (ICs) which have been influenced with satellite data. This investigation quantifies the degree of improvement that the CRAS-produced ICs have in WRF simulations out to 36 hours during portions of the Northern Hemisphere fall months of September and October 2011, where there are a combination of both moist and dry regimes over the north central United States.

This paper will provide a summary of the current state of GOES Sounder radiance and retrieval assimilation in numerical models as a motivating factor for this research; the design of the experiment in seeking to quantify the impact of these retrievals on a regional-scale domain; and some results and comparisons between the WRF simulations, CRAS, and validating analyses and point observations for certain moisture fields. The objective is to develop a methodology for an effective, applicable study easily replicated in the field that confronts substantial forecast problems resulting from tropospheric moisture gradients which are inadequately resolved in NWP guidance at the current time.

\section{Background of Problem}

The basic premise of NWP is that it is an initial value problem. In striving to attain the perfect forecast, there are several other factors which constrain the accuracy of the solution, including parameterizations and approximations within the model; schemes which use time-stepping to solve partial differential equations over a finite interval; atmospheric features occurring on spatial and temporal scales smaller than resolved by the model; limited observations to populate the initial analysis, particularly above the surface and away from land; the quality and accuracy 
of those observations and the representation of any observation errors during the assimilation process; and the boundary conditions on the perimeter of the domain which can force the solution for long-duration simulations. The United States' National Centers for Environmental Prediction (NCEP) operational models use numerous data sets consisting of in-situ and remotely sensed observations in building their analysis. However, some forecasters have indicated that moisture representation in the NCEP models is sometimes inadequate for forecasting mesoscale precipitation events (G. Mann 2011, personal communication).

To resolve this issue, additional moisture information was sought from unexploited earthobserving satellite instruments for incorporation into model simulations. Retrievals from the GOES-13 Sounder were chosen due to the limited amount of use during the current assimilation process in the NCEP operational models (Keyser et al. 2011). As of this writing, the North American Mesoscale (NAM; http://www.emc.ncep.noaa.gov/?branch=NAM) model and Global Forecast System (GFS; http://www.emc.ncep.noaa.gov/GFS/) model do use brightness temperatures from the GOES Sounders (GOES-11 and GOES-13) over ocean as part of their radiance assimilation system. However, they do not use retrievals, nor do not use the GOES Sounder observations over land. The Rapid Update Cycle (RUC) model, which is transitioning to the Rapid Refresh (RR; http://rapidrefresh.noaa.gov/) model, does use PW retrievals, but only those over ocean from the GOES-11 Sounder.

Conservation of enthalpy. Theory provides a connection between temperature and moisture during convective processes. As convective towers ascend, the parcel cools and condenses resulting in a release of latent heat. In order to conserve moist enthalpy $\mathrm{E}$,

$$
E=C_{p} T+L_{v} q \text {, }
$$

where $C_{p}$ is the heat capacity at constant pressure, $T$ is temperature, $L_{v}$ is the latent heat of vaporization, and $q$ is specific humidity, not only does the convection require a removal of water vapor from the parcel, but that amount must be directly proportional to the change in temperature. This relationship must also hold for the depth of the convective cloud, from the base pressure at the LCL, $C_{\text {base }}$, to its top pressure at the equilibrium level, $C_{\text {top }}$, as shown in Baldwin et al. (2002), such that:

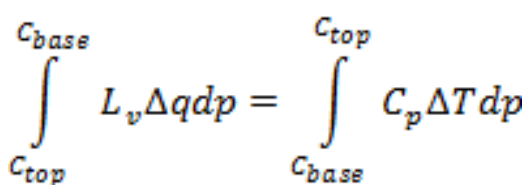

Therefore, the availability of middle and upper tropospheric moisture for deep convective processes is a factor in their strength, effectiveness, and longevity, because as environmental temperature increases aloft, the stability increases and parcel buoyancy decreases. In fact, it has been demonstrated that tropical convection is respondent to mid-level moisture, as found in Shepherd et al. (2001) and Thompkins (2001). This is also an observation which has been incorporated into the Kain-Fritsch (KF) convective scheme (Kain 2004), and further confirmed by Knupp and Cotton (1985), which found that environmental humidity is an important factor in assessing downdraft strength. The KF scheme is the convective parameterization of choice in this study to allow the model solution to indicate increased sensitivity as a consequence of differential moisture resulting in vertical mass fluxes. 
Convective scheme. The WRF simulations in this experiment all utilize the KF convective parameterization, which is a mass flux scheme, and thus requires an adjusted response based on the grid scaling. The closure for the KF scheme is convective available potential energy (CAPE). This is an important source for latent heat release, and thus, accumulated convective precipitation. It has been shown in Kain and Fritsch (1990) that the normalized vertical mass flux varies significantly - by a factor of two - in the upper troposphere for changes of relative humidity between $50 \%$ and $90 \%$. This sensitivity is critical because, for cold temperatures, the amount of water vapor mixing ratio required to adjust the relative humidity is not particularly substantial.

\section{Data and Methodology}

The configuration of the models in this WRF transition experiment was intended to be easily duplicated in the field as part of the EMS. The dynamics core and physics packages chosen for the ARW runs closely match those from the local WRF model used at that NWS office in Milwaukee, Wisconsin. Table 1 outlines the core configuration. The WRF code used for simulations throughout the study was version 3.1.1.

\begin{tabular}{|l|l|}
\hline Dynamics & Non-Hydrostatic \\
\hline Cumulus Scheme & Kain-Fritsch \\
\hline Microphysics Scheme & WSM Single-Moment 5-Class \\
\hline PBL Scheme & Yonsei University \\
\hline Land Surface Scheme & NOAH \\
\hline Surface Layer Physics & $\begin{array}{l}\text { Monin-Obukhov with heat and } \\
\text { moisture surface fluxes }\end{array}$ \\
\hline Long Wave Radiation & RRTM \\
\hline Short Wave Radiation & Dudhia Scheme \\
\hline Time-Integration Scheme & Runge-Kutta $3^{\text {rd }}$ Order \\
\hline Damping & Rayleigh \\
\hline
\end{tabular}

TABLE 1. The core configuration for the Weather Research and Forecasting (WRF) model used in the experiment. The dynamical package was the Advanced Research WRF (ARW). Each simulation had an adaptive time step. References for the schemes can be found in the Skamarock et al. (2008) technical note.

The domain selected for the simulations was over the north central United States, including the Northern Plains and Upper Mississippi Valley. The Lambert Conformal grid contained 100 grid points in each horizontal direction with equal-area spacing of $20 \mathrm{~km}$ to minimize the time to complete each simulation. The domain was thus square with $2000 \mathrm{~km}$ on each boundary and 45 vertical levels up to a model top of $50 \mathrm{hPa}$. The WRF model runs were initialized twice daily during the experiment period at the standard times of 00 and 12 UTC and executed out to 36 hours, outputting every hour.

Configuration of control and experimental WRF runs. There was one control and two experimental WRF runs, all of which utilized a MODIS sea surface temperature composite for water grid points (Haines et al. 2007) and soil moisture from the operational 0.5-degree GFS distributed by NCEP. Soil temperatures and the source of atmospheric properties were different based on the run. Temperature, wind, relative humidity (water vapor mixing ratio), cloud water mixing ratio, geopotential height, and surface pressure were pre-processed by the WRF prior to each unique model simulation. Additional moisture information was available for the first experimental run (WRFY). As part of the WRF preparation process, input model fields were 
interpolated both vertically and horizontally to the WRF grid, which resulted in some minor smoothing to the analysis.

The control run, herein referred to as WRFX, contained ICs and boundary conditions (BCs) from the GFS executed six hours prior to the experiment initialization time. Thus, the six-hour forecast from the GFS was used to initialize the WRFX run. Lateral boundaries were forced every three hours from the same GFS run. Moisture components of the GFS initial and boundary conditions were relative humidity and cloud water mixing ratio.

The first experimental run, herein referred to as WRFY, contained ICs and BCs from a CRAS simulation run on an expanded $45 \mathrm{~km}$ grid with an identical projection, allowing lateral boundaries to be forced hourly. Moisture initialization in the WRFY came from four mixing ratios produced by the CRAS pre-forecast procedure: water vapor, cloud water, ice water, and rain water. The CRAS utilized only one cloud mixing ratio and one precipitation mixing ratio, however. The form of the water carried in the CRAS mixing ratio arrays was a function of the temperature. They were classified prior to being served to the WRF preparation process as input. The background for the CRAS simulation was the same GFS run as used in the WRFX run.

The second experimental run, herein referred to as WRFZ, used the ICs from a "cold start" CRAS initial-hour assimilation but BCs from the previous GFS run, as in the WRFX. Like the WRFX, the WRFZ used a six-hour forecast from the GFS as the IC background. The WRFY took advantage of the CRAS pre-forecast assimilation of GOES Sounder retrievals into the ICs, which is commonly known as a "hot start". The WRFZ took advantage of the Sounder retrievals which improve the moisture analysis only at the initialization time. Moisture initialization in the WRFZ came in the form of water vapor mixing ratio only.

The purpose and configuration of the WRFZ run was strictly to assess whether the updated moisture analysis would have an impact on short-term forecasts of moisture-related variables: relative humidity, total precipitable water (TPW), and accumulated precipitation. In contrast, the intent of the WRFY experiment was to see whether the CRAS could build an analysis which could outperform a simple assimilation technique, or no assimilation at all, since the CRAS was developed to assess the impact of space-based observations on the accuracy of NWP solutions. Producing forecasts using parameterizations and techniques which exploit and give merit to information from satellites, particularly the GOES Sounder, was thus a necessary component of the project.

\section{Precipitable Water Results}

In assessing the value of the GOES-13 Sounder retrievals to the WRF forecasts, it was first necessary to examine the improvement to the PW analyses initializing the model runs. The subsequent evaluation focused on the impact of the retrievals on forecasts, in 12-hour increments, through 36 hours, for each of the simulations, the control and both experiments. TPW was compared against the NAM analysis and point Global Positioning System (GPS) integrated precipitable water (IPW) observations. For comparisons involving NAM mass fields (TPW), statistics were computed for grid points within the interior of the domain; for those involving the NDFD, statistics were computed for grid points within the continental United States in the interior of the domain; for those involving GPS-IPW, statistics were computed for TPW at grid points near the observation sites. Interior grid points were used for the grid 
verification of the model domain instead of all eligible grid points to discount any BC and WRF blend zone influences from the results. The Model Evaluation Tools (MET) package, version 3 (http://www.dtcenter.org/met/users/), was used to compute the statistics. The primary statistics used to assess performance were mean absolute error (MAE) and root-mean-square error (RMSE).

The evaluation period consisted of 21 times between 00 UTC on 28 September 2011 and 00 UTC on 8 October 2011. This period was chosen for both dry and moist regimes. In addition, most precipitation was the result of well-forced, kinematic processes rather than thermodynamically driven. The objective was to establish whether a remotely sensed, integrated quantity could be adequately analyzed to a three-dimensional grid and produce favorable results in short-term forecasts, under 36 hours.

Error of experiment and control WRF analyses compared to GPS-IPW. An initial investigation calculated the mean TPW MAE for the experimental runs compared against GPSIPW during the evaluation period. There was not a discernible leader. The difference in mean MAE over this period between the best performer, the WRFX (control), and the WRFY was 0.03 mm. The WRFY had the lowest MAE for $38 \%$ of the 21 periods; the WRFZ had the lowest MAE for 33\% of the periods; and the WRFX had the lowest MAE for $28 \%$ of the periods, which also indicates a similar trend in the run-to-run RMSE during the experiment period. The inconclusive results were likely due to the poor spatial heterogeneity of GPS-IPW sites across the domain compared to the magnitude of the correction. Because of this, it is possible that some horizontal moisture distributions favor one analysis over the others.

\begin{tabular}{|l|l|l|l|}
\hline GPS-IPW & WRFX-00 & WRFY-00 & WRFZ-00 \\
\hline MAE $(\mathrm{mm})$ & 1.58 & 1.61 & 1.59 \\
\hline RMSE $(\mathrm{mm})$ & 2.07 & 2.11 & 2.10 \\
\hline
\end{tabular}

Error of experimental and control WRF 12-hour forecasts compared to GPS-IPW. The aforementioned case was representative of the mean quantitative results over the experiment period. Using GPS-IPW point observations, the 12-hour forecast comparison indicated that the WRFZ narrowly outperformed the WRFX with a lesser mean MAE for TPW by $0.05 \mathrm{~mm}$. For both the 24-hour and 36-hour forecasts, the WRFZ and WRFX performed statistically about the same, with a mean MAE difference no greater than $0.01 \mathrm{~mm}$. During this period, the TPW MAE was lowest for the WRFZ 12-hour forecast nine of 21 times, or 43\%. This was in comparison to the WRFX, which had the lowest MAE only five of 21 times, or $24 \%$. The WRFY had the lowest MAE seven of 21 times, or 33\%. This indicates that the WRFY analysis was competitive, but occasionally exhibited a higher MAE for individual misses than the WRFX and WRFZ, which increased its mean TPW MAE. Of particular interest is that the WRFY analyses exhibited a lower TPW MAE for four of the last five periods evaluated. The 12-hour forecast TPW MAE for the WRFY was likewise the lowest for four of the last five periods. This lends credence to the already seminal holding that NWP is an initial-value problem and more accurate ICs result in a more accurate forecast. 


\begin{tabular}{|l|l|l|l|}
\hline GPS-IPW & WRFX-12 & WRFY-12 & WRFZ-12 \\
\hline MAE (mm) & 1.77 & 1.81 & 1.72 \\
\hline RMSE (mm) & 2.27 & 2.37 & 2.24 \\
\hline
\end{tabular}

Error of experiment and control WRF 12-hour forecasts compared to NAM analysis. In order to confirm the result of the point comparison, a grid analysis comparison was arranged. The grid comparison used the NAM analysis of TPW, which contains the GPS-IPW observations as input. All grid points within the verification area were compared. The verification grid and forecast grid were collocated after re-projecting the verification analysis. This was completed through remapping and upscaling the NAM analysis from its native projection and resolution to that of the experiment domain. A bilinear interpolation was used as part of the subsampling. WRF model output was not re-projected. The mean TPW MAE calculated with this approach over the experiment period reached the same result as the GPSIPW point-wise comparison. Using the NAM analysis and comparing all verification grid points, the WRFZ mean MAE was $0.04 \mathrm{~mm}$ less than the WRFX, which produced the next lowest mean MAE. The trend throughout the evaluation period can be found in Figure 1. The WRFY exhibited the worst mean MAE, but that was only $0.16 \mathrm{~mm}$ greater than the WRFZ.

\begin{tabular}{|l|l|l|l|}
\hline NAM & WRFX-12 & WRFY-12 & WRFZ-12 \\
\hline MAE (mm) & 1.97 & 2.09 & 1.93 \\
\hline RMSE (mm) & 2.59 & 2.78 & 2.56 \\
\hline
\end{tabular}

The performance of the WRFX and WRFZ at the 24 and 36 hour forecasts were not discernible. Again, the difference in the mean MAE was $0.01 \mathrm{~mm}$ or less between the two for both forecast hours. The WRFY had a higher mean MAE at those forecast hours, but still indicated some strength relative to the WRFX and WRFZ during the last five periods.

\section{Conclusions}

This study was an initial investigation into the benefits of using GOES-13 Sounder retrievals as part of regional NWP to improve forecasts of TPW. In the evaluations conducted here, the retrievals were found to be inconsequential in many cases and did not produce a consistent positive reflection in the statistics. This indicates that instruments onboard our earth-observing geostationary satellites need spectral improvement to supply a meaningful correction to analyses used in regional NWP. It also requires a reassessment of how the operational NWP community uses indirect moisture information from remote sources beyond the techniques explored here. While the results presented are perhaps a testament to the adequacy of current analyses, they stand as a challenge to improve numerical techniques for assimilating additional data because the number of data sets assimilated into operational models continues to grow. Yet, NWP solutions 
are far from perfect and, as demonstrated, there is information from the GOES Sounder which can improve moisture representation in models and alter forecasts for the better.
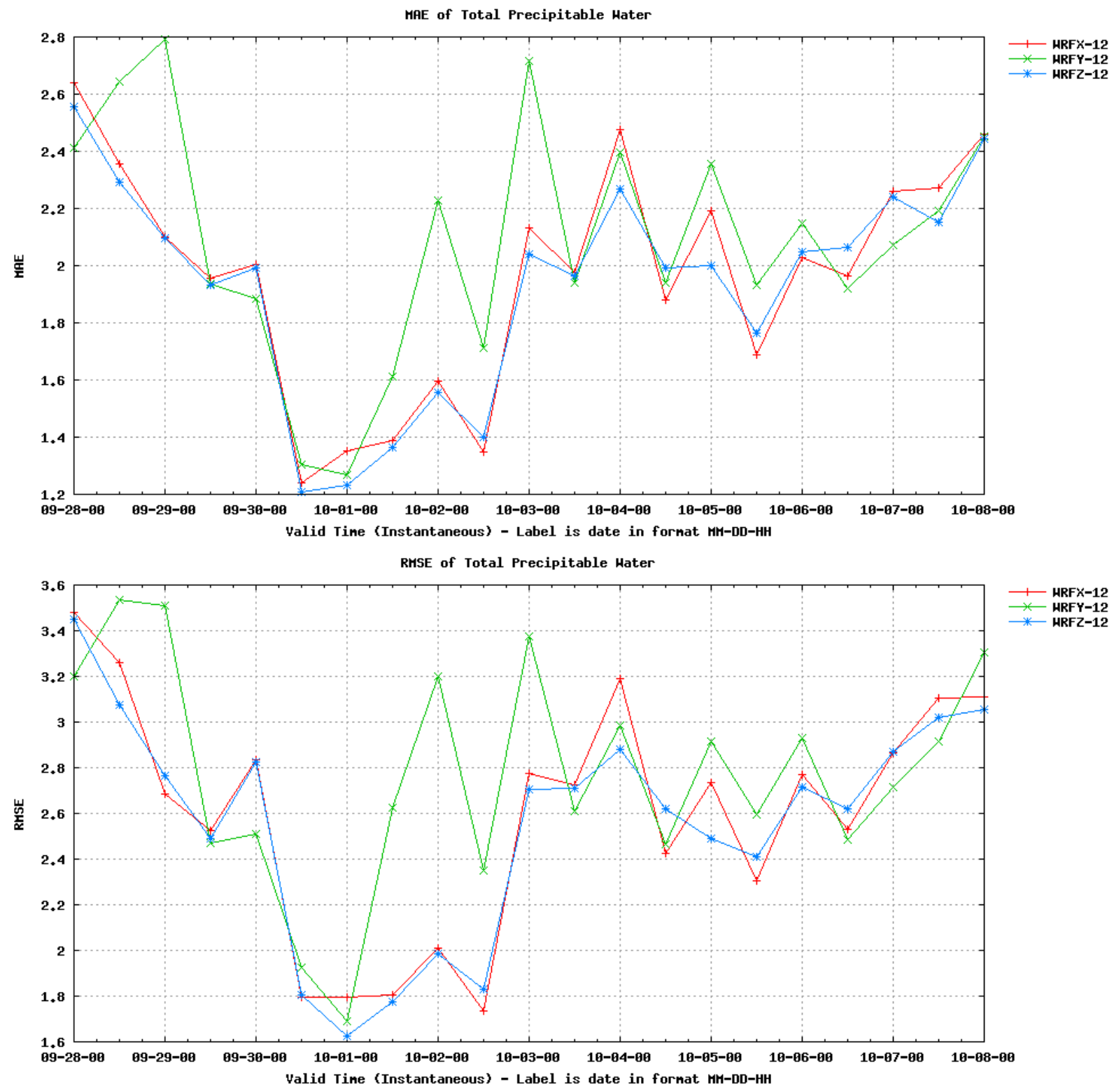

FIG. 1. Mean absolute error (top) and root-mean-square error (bottom) for total precipitable water (mm) over the period from 00 UTC 28 September 2011 to 00 UTC 8 October 2011. Error is calculated based on the NAM analysis at the valid time compared to the 12-hour forecasts of the WRFX (red), WRFY (green), and WRFZ (blue) for the same time. Note the change to the scale on the left ordinate axis between the figures.

Benefits were expected in part due to a casting of the ARW governing equations in flux form in conjunction with the KF convective parameterization, which is sensitive to variations in middle 
and upper tropospheric relative humidity as part of its formulation. The method and results were tempered based on the limited vertical resolution of the GOES Sounder. During the summer months, the GOES Sounder water vapor channels are most capable of detecting temperature and moisture gradients in the upper troposphere, and sense relatively little, if any, boundary layer moisture. A one-dimensional variational assimilation scheme was used to add or subtract water vapor from a model sounding, indiscriminately of vertical gradients, based on three sigmabounded layers produced by the Li et al. (2008) enhancements to the retrieval process.

Comparing WRFX and WRFZ, two sources of PW verification confirmed forecasts were slightly better 12 hours after initialization if GOES-13 Sounder input was included. Results were calculated based on the GPS-IPW network and confirmed using a NAM analysis. There was no substantial impact of the added observations at 24 or 36 hours in the flow regime of the period studied from late September into early October. While the WRFY had fleeting success, the indications were that the CRAS dynamics and physics were controlling and negatively influencing the solution, even at short intervals from the initialization time.

Thus, in order for an accurate forecast of cloud, water vapor, and precipitation distributions, it is necessary for our NWP models to contain a detailed and accurate analysis of moisture. The current NCEP operational models are good, but there remains a small margin for improvement from assimilating additional observations if done so with skill and knowledge of the dynamics and parameterizations within the model that would respond to such changes in producing a forecast. At the current time, this is only possible through the use of satellite products. Using the CRAS pre-forecast and assimilation techniques in conjunction with the WRF has allowed for GOES Sounder observations in the form of retrievals to impact the solution. The WRF transition experiment conducted during the early fall of 2011 has been able to better quantify the degree of this effect, and will continue in real-time for upcoming seasons. While the results are tempered by some inherent shortcomings in the capabilities of the instrument, assimilation scheme, and numerical model, the strategy and path forward are clear. A delicate investigation of moisture integration techniques within assimilation constraints and model parameterizations for different seasons and flow regimes can slowly extract gainful information from the current, and future, geostationary platforms.

\section{References}

Aune, R. M., 1994: Improved precipitation predictions using total precipitable water from VAS. Preprints, 10th Conference on Numerical Weather Prediction, American Meteorological Society, Portland, OR, $192-194$.

Haines, S. L., G. J. Jedlovec, and S. M. Lazarus, 2007: A MODIS sea surface temperature composite for regional applications. IEEE Trans. Geosci. Remote Sens., 45, 2919-2927.

Kain, J. S. and J. M. Fritsch, 1990: A one-dimensional entraining/detraining plume model and its application in convective parameterization. J. Atmos. Sci., 47, 2784-2802.

Kain, J. S., 2004: The Kain-Fritsch convective parameterization: an update. J. Appl. Meteor., 43, $170-181$.

Keyser, D., Environmental Modeling Center, National Weather Service, cited 2011: PREPBUFR Processing at NCEP.

[Available online http://www.emc.ncep.noaa.gov/mmb/data_processing/prepbufr.doc/document.htm.]

Knupp, K. R. and W. R. Cotton, 1985: Convective cloud downdraft structure: an interpretive survey. Rev. Geophys., 23, 183-215.

Li, Z., J. Li, W. P. Menzel, T. J. Schmit, J. P. Nelson, III, J. Daniels, and S. A. Ackerman, 2008: GOES sounding improvement and applications to severe storm nowcasting, Geophys. Res. Lett., 35, L03806. 
Shepherd, J. M., B. S. Ferrier, P. S. Ray, 2001: Rainfall morphology in Florida convergence zones: a numerical study. Mon. Wea. Rev., 129, 177-197.

Skamarock, W. C., J. B. Klemp, J. Dudhia, D. O. Gill, D. M. Barker, W. Wang and J. G. Powers, 2008: A description of the Advanced Research WRF version 3. NCAR Technical Note, TN-468+STR, 113 pp.

Tompkins, A. M., 2001: Organization of tropical convection in low vertical wind shears: the role of water vapor. $J$. Atmos. Sci., 58, 529-545. 